\title{
LOGIC COMPRESSION OF DICTIONARIES FOR MULTILINGUAL SPELLING CHECKERS
}

\author{
Boubaker MEDDISB IIAMROUNI
}

\author{
GETA, IMAG-campus (UJF \& CNRS) \\ BP 53, F-38041 Grenoble Cedex 09, FR $\wedge$ NCI: \\ Boubaker. Meddeb-Hamrounie imag. fr
}

\begin{abstract}
To provide practical spelling checkers on micro-computers, good compression algorithms are essential. Current techniques used to compress lexicons for indo-liuropean languages provide efficient spelling checker. Applying the same methods to languages which have a different morphological system (Arabic, Turkish,...) gives insuflicient results. "To get better results, we apply other "Iogical" compression mechanisms based on the structure of the language itself. Experiments with multilingual dictionaries show a significant reduction rate attributable to our logic compression alone and even better results when using our method in conjunction with existing methoxls.
\end{abstract}

KEY WORDS: Spelling checkers, Multilinguism, Compression, Dictionary, Finite-state machines.

\section{INTRODUCTION}

Since the first work in 1957 by Glantz [6], a greal deal of theorizing and research has taken place on the subject of spelling verification and correction. Many commercial products (word processors, desktop presentation,...) include eflicient spelling checkers on micro-computers. 'The classical methods, used are generally based on a morphological analyzer. This is sufficient to provide a robust monolingual spelling checker, but using morphological analyzers can become unrealistic when we want to develop an universal solution. In fact, the analyzers built for each language use various linguistic models and engines, and it is impossible to convert a morphological analyzer from one formalism to another. Furthermore, using these classical methods would lead to combining into the host application as many of grammars and parsers as languages, which would increase the code size and the maintenance problem of rules and data. The method presented in this paper is based on building a dictionary of all surface forms for each language, which is sufficient for spelling checkers applications. The dictionary built with the existing generattors can be casily updated manually but may be huge, especially for some agglutinative language (Arabic, T'urkish,...). $\wedge$ compression process on the multilingual dictionaries is necessary to obtain a reduced size. The existing compression methols generally used are physical and provide good results for indo-European languages. Applying the same techniques to other languages ( $A$ rabic, Turkish,...) shows their limits. For this reason we introduce a new kind of compression techniques that we called "logic compression". This new technique requires a primitive morphological knowledge during the compression process and requires less stotage space than previous methods. It also has the advantage of being an universal method applicable to all languages.

Section 1 contains an overview of existing methods for building spell checkers and the limits of such system
$\&$

WinSoft SA.

34, Bd. de l'Tsplanade

I:-38000 Grenoble, IRANCL:

when we take into account new constraints such as multilingualism. Section 2 outlines the lirst two steps of our work: we adapt an existing method to Arabic, then make a first extension by introducing a new kind of compression called "logic compression". Section 3 introduces in detail the logic compression with its application to other languages, and shows the improvements obtained when using logic compression in conjunction with existing methods. Section 4 outlines the architecture of our multilingual spelling checker system and some future projects.

\section{OVERVIEW OF EXISTING METHODS}

\section{I.1. Grammar-based approach}

These methods were used in the beginning on early computers when storage space was expensive. It consists in building a small lexicon containing roots and aflixes, a granmar of rules that express the morphographemic altermations, and an engine that uses the grammar and the lexicon to see if an input word belongs to the language or not. If the process of recognition fails, some operations (substitution, insertion,...) are performed on the misspelled word to provide a list of candidate words that helps the user to select the eorrect form.

leven though, it is a great accomplishment to design a powerful engine [3] [8] and to express rules in a pseudo natural way [9] even for different languages [1] [2] [1], these systems present some limits:

- Multilinguism: 'This methods does not support all langnages. To offer a multilingual solution for in languages you have to store n grammars and n lexicons, and generally $n$ different engines into the host application.

- Cost of retrieval: For some languages, the retrieval of words may be long. I'or instance, a vocalized Arabic spell checker must accept non-vocalized or partially vocalized words which require more time to be acepted than fully vocalized words.

- Cost of guessing alternatives for a misspelled word: 'lo guess a correct word when a misspelied word is found, we have to modify the misspelled word by all possible operations (substitution, insertion, suppression,...) for 1 or 2 characters and then try to check them. This matter can take a lot of time before displaying the correct forms for endusers.

- Maintaining the grammars and data: The grammars and lexicon require continuous updating. You need to find a multilingual computational linguist who knows the linguistic theory and the formalism to easily update data and rules [8].

- Ergonomic fealures: In some languages, end users want to have some options that let them choose how the spell checker will accept words. In Arabic, for example, different. regions have slightly different orthographical conventions. 


\section{I.2. Iexical-based approach:}

Lexical-based approach appear after the first methods described above, when storage space become less expensive. 'The first step is to build complete list of surface forms belonging to the language using morphological generators, SIJP (Specialized languages for Linguistic Programs), etc. and then compresses the large word-dictionary. They are generally used for office applications such as word processors, desktop presentation, etc. Their main advantage is that they cover a complete language since all the forms can be found in the initial list. Also, they allow efficient retrieval and guessing of misspelled words [4]. Irowever, some limits exist in such systems:

- Multilinguism: The compression process give a good ratio for languages with a weak inflexion factor (Inglish,...) where the compression mechanism give up to $150 \mathrm{~KB}$ of storage from around $3 \mathrm{MB}$ of a full list [4]. "The compression technologies are still powerful for languages with a medium inflexion factor (Russian,...). For example, a list of all surface Russian words of between 10 and 15 MB of size can be reduced to $700 \mathrm{~KB}$ [4]. For languages with a high inflexion factor (Arabic, Finnish, Ilungarian,...), it won't be easy to find compression technologies that give practical results [4]. For instance, a lull list of completed vocalized words in Arabic has $300 \mathrm{MB}$ in size and the current compression methods are impraclical.

- No morphological knowledge: These methods are nentral with respect to the text language, the efficiency of compression techniques may be improved by using specific properties of the language [4].

\section{A FIRST APPROACH: ADAPTING AN EXISTING METHOD FOR $\triangle$ RABIC}

\section{II.1. Using an existing method}

As a first step, we take an efficient method used 10 compress dictionaries for European (inglish, Fiench,...) spelling checkers [4] and try to apply it to Arabic. 'The first step of our work consists in building a full list of surface forms using a morphological generator [5] and completed by all irregular forms and existing corpus. The final large word dictionary which covers non-vocalized Arabic has a size of $75 \mathrm{MB}$. The compression process yieks 18 MB in a compressed format. Ior an idea of the compression process readers can refer to [10]. Table 1 gives somc results of the compression process for a few Iuropenn languages to see the efficiency of the methox and its inakequacy for the Arabic language.

\begin{tabular}{|c|c|c|c|}
\cline { 2 - 4 } \multicolumn{1}{c|}{} & $\begin{array}{c}\text { word } \\
\text { forms }\end{array}$ & $\begin{array}{c}\text { size } \\
\text { uncompressed }\end{array}$ & $\begin{array}{c}\text { size } \\
\text { comprescel }\end{array}$ \\
\hline Danish & 448.000 & $5689 \mathrm{~KB}$ & $725 \mathrm{~KB}$ \\
\hline Cremant & 403.000 & $5297 \mathrm{~KB}$ & $866 \mathrm{~K} 13$ \\
\hline Arabic & 7 millions & $75 \mathrm{MB}$ & $18 \mathrm{M} 13$ \\
\hline Inglish & 88.000 & $841 \mathrm{~KB}$ & $224 \mathrm{Kl}$ \\
\hline
\end{tabular}

The result for Arabic is impractical for small computers. We must then find other techniques that produce a smaller dictionary or extend this method; to get an exploitable solution.

\section{II.2. Extension of the method:}

The initial idea is applied to the morphological system of Arabic. While most of the fully inflected forms words in Arabic are built by addling to a stem prefixes and suffixes we propose replacing some words with only one form beginning by a special code that represents a family of prefixes and finishing by another special code which represents a family of suffixes. For this purpose, we wrote a program in MPW-C that processes a finll list of inflected forms and using an existing decomposition of alfixes into sub-sets already established, give the reduced lexicon where many forms ate replaced by only one representation $\left(12 S_{i}{ }_{2}\right.$ stem $S_{-} S_{j}$ ) where $P S_{i}$ (with respect to $S S_{j}$ ) is the set $i$ (with respect $10 \mathrm{j}$ ) of prefixes (with respect to sulfixes). Note that the reduced lexicon represents laithfully the initial list without any silence (missing words) or noise (incorrect words). Only compressed words are replaced, and the rest remain in the reduced list. The ligure 1 gives an example of words, an example of a decompositions and the obtained result.

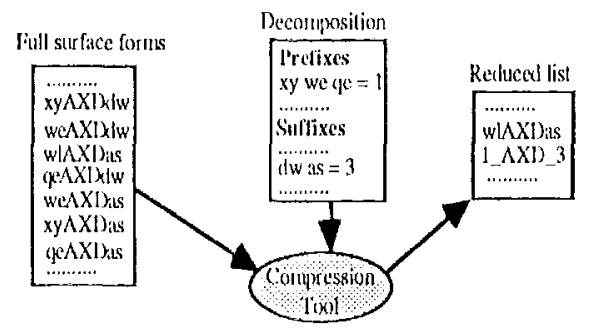

lig. 1: lixample of the compression process

The next crucial problem to resolve is 10 find the best decomposition that provide the best reduced lexicon. The methorl must be antomatic. It must process the large worddictionary, and regarding an initial list of prelixes and suffixes, must give as output the best decomposition and the optimal reduced dictionaty. But, before studying the inplementation of such an algorithm, we began, to sec how much space we could gain by this technique stanting from a manual decomposition.

- Mannal method: Starting from a different full lists for each category of words (transitive verbs, nouns,...), we choose different decompositions and processed the full list with the compression tool. The best decomposition kept for each category was the decomposition which eliminated the maximum forms. 'This method gave many candiclate decompositions depending on the grammatical category of the word. To choose the best globil one we took into atecount the frequency of dictionary entries. This method was tested on different Arabic word lists and some results are described here. Readers can refer 10 [10] or [11] for more information. To sec some decomposition, consider the following sets:

$l_{1}=\{w a, f a\}, 1,4 / \quad i_{2}=\{l a, s a\}, / l_{j} /$

$\mathrm{li}_{3}=[\mathrm{nia}, \mathrm{iti}\}, \mathrm{j} \mathrm{i} / \ldots \ldots$.

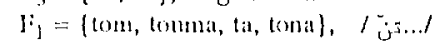

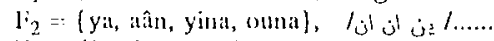

$\mathrm{F}_{6}=$ (hat, hâ, ya, ka, kom, kouma, kona, hom, houna, homa, natî $\}, E_{7}=V_{6} \backslash\{y a, n a \hat{i}\}+\{n i\}$,

$I_{9}=\{$ wa $\}, \ldots .$.

$\mathrm{L}_{\mathrm{i}}$ (with respect to $F_{\mathrm{j}}$ ) is a set of prelixes (with respect to sulfixes). We note the quantity $l_{i}, I_{j}$ (wilh respect to $\mathrm{I}_{\mathrm{i}}, \mathrm{I}_{\mathrm{j}}$ ) all strings built by a concatenation of each element of $\mathrm{P}_{\mathrm{i}}$ (with respect to $l_{i}$ ) with each element of $E_{j}$ (with respect $10\left(i_{j}\right)$.

lixample of 3 class (from 6) of the prelix class: 


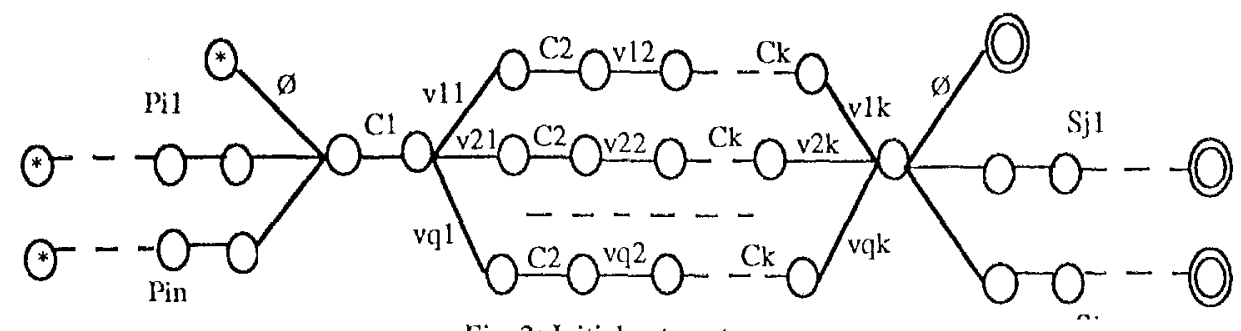

Fig. 2: Initial automaton

$P_{1}=E_{1} \cdot P_{2}=E_{4}$.

$P_{3}=E_{3}+E_{2} \cdot E_{3}+E_{1} \cdot E_{2} \cdot E_{3}$

Example of 4 class (from 13) of the suffix class:

$S_{1}=F_{1} . S_{2}=F_{2} . S_{7}=F_{7} . S_{8}=F_{9} \cdot F_{7}$.

- First results: case of Arabic; With all the classes already found for Arabic (6 classes of prefixes, 13 classes of suffixes; each class containing an average of 8 affixes), we processed a collection of non-vocalized Arabic dictionarics (17 MB), the result gave a reduction lexicon of $254 \mathrm{~KB}$. Used this in combination with the compression process described in $\$ 1.2$, the final result is $121 \mathrm{~KB}$. Note also that part of this work was implemented in a commercial multilingual word processor $\left(W_{i n} \Gamma^{\circ} t^{\circ}\right)$ to ofler Arabic spell checking.

\section{LOGIC COMPRESSION:}

\section{III.1. Theoretical aspects:}

Let $\mathrm{V}$ be a finite set and $\mathrm{V}^{*}$ the set of words built on $V$ including null strings noted $\emptyset$.

$W \in V^{*} . W=W_{1} W_{2} \ldots W_{n} . W_{i} \in V$.

$i \in[1 . . n]$. Let $V^{+}=V^{*}-\{\emptyset\}$.

Let $Y$ be a sub-set of $V$ that contain vowels.

1. Prefix(W). $\forall W \in V^{+}$.

We call order $i$ prefix the quantity:

$P_{i}=W_{1} W_{2} \ldots W_{i} . \quad(1 \leq i \leq n-1)$.

2. $\operatorname{Suffix}(W) . \quad \forall W \in V^{+}$.

We call order $j$ suffix the quantity:

$S_{j}=W_{j} W_{j+l} \ldots W_{n} . \quad(1 \leq j \leq n)$.

3. $\operatorname{VocPat}(W) \quad \forall W \in V^{+}$.

We call vocalic pattern of $W$ the set:

$V y=\left\{W_{i}, W_{j}, \ldots W_{k}\right\} . \quad W_{i} \in Y$.

$\operatorname{card}(V y) \leq$ length $(W)$

4. $R \cot (W) . \quad \forall W \in \mathrm{V}^{+}$.

We call root the quantity:

$\mathrm{R}=\mathrm{W}_{\mathrm{p}} \ldots \mathrm{W}_{\mathrm{q}} .(1 \leq \mathrm{p}<\mathrm{q} \leq \mathrm{n})$,

$\operatorname{card}(R) \leq q-p+1$.

5. $P_{i}$ : Prefixes class. $P_{i}=\left\{\varnothing, P_{i 1}, P_{i 2}, \ldots P_{i k}\right\}$.

$P_{i j}$ is a prefix. $\quad 1 \leq \mathrm{j} \leq \mathrm{k}$

$\operatorname{Card}\left(P_{i}\right)=k+1 . \quad$ if $k \geq 1$.

$$
=1 \text {. if } P_{i}=\{\varnothing\} \text {. }
$$

6. $S_{j}$ : Suffixes class. $S_{j}=\left\{\emptyset, S_{j 1}, S_{j 2}, \ldots S_{j k}\right\}$.

$S_{\mathrm{ji}}$ is a suffix. $\quad 1 \leq \mathrm{i} \leq \mathrm{k}$

$\operatorname{Card}\left(S_{j}\right)=k+1 . \quad$ if $k \geq 1$.

$$
=1 \text {. if } S_{j}=\{\varnothing\} \text {. }
$$

7. $\mathrm{V}_{\mathrm{k}}$ : Vowel class.

$V_{k}=\left\{\varnothing, V y_{k 1}, V y_{k 2}, \ldots V y_{k k}\right\}$
$\mathrm{V}_{\mathrm{ki}}$ is a vocalic pattern. $1 \leq \mathrm{i} \leq \mathrm{k}$

$\operatorname{Card}\left(V_{k}\right)=k+1 . \quad$ if $k \geq 1$.

$$
=1 . \quad \text { if } \mathrm{V}_{\mathrm{k}}=\{\emptyset\} \text {. }
$$

III.2. Logic Compression: What is it ?

Let's take the following automata that represent some surface vocalized words (lig 2)

$P_{i j}$ is a prefix. $1 \leq \mathrm{j} \leq \mathrm{n}$.

$\mathrm{S}_{\mathrm{ji}}$ is a suffix. $1 \leq \mathrm{i} \leq \mathrm{n}$.

$C_{j}$ are the consonants of the vocabulary.

$1 \leq \mathrm{i} \leq \mathrm{k}$.

$v_{i j}$ is the vowel attached to the consonant $C_{j}$.

$\mathrm{l} \leq \mathrm{i} \leq \mathrm{q}$ and $\mathrm{l} \leq \mathrm{j} \leq \mathrm{k}$.

$\varnothing$ is the null string.

This automata recognizes all words beginning from an initial state (marked by *) and finishing in a final state (marked by a double circle)

The number of arcs of such an automata is:

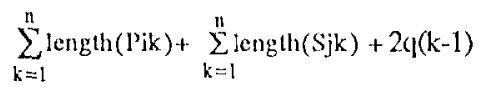

If we consider, for example, that affixes have a single character, the number of ares is cqual to $2(n+1)+2 q(k-1)$.

The logic compression consist in supplying the class of prelixes, suffixes and vowels and replaces each set by only one are that represent a family of prefixes, suffixes or vowels.

Starting from the following sets already established: $P_{i}=\left\{\left(Q_{1}, P_{i 1}, P_{i 2}, \ldots P_{i n}\right\}\right.$ a class of prelixes stored as $x$.

$S_{j}=\left\{\emptyset, S_{j 1}, S_{j 2}, \ldots S_{j 11}\right\}$ a class of suflixes stored at $y$.

$v_{k}=\left\{\left(v_{11} \ldots v_{1 k}\right\},\left(v_{21}, \ldots v_{2 k}\right\}, \ldots\left(v_{1 \mid 1}, \ldots v_{1 \mid k}\right)\right\}$ a clatss of vecalic pattern stored as $z$.

The logic compression reduces the intial automaton to this new one:

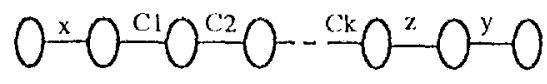

Fig. 3: Reduced automata

The number of ares kept in the automata is equal to $3+k$. The set $V_{k}$ contains a sub-set of $k$ vowels which must be applied to the last $k$ characters.

Irr.3. Lxperiments:

The logic compression with only an alfix decomposition, built by the manual method explained above, hats been tested on various list of words that represent collections of multilingual diclionaries (a list of inflected forms). Three languages are tested: non-vocalized Arabic which has a great intlexion factor, French which has a 


\begin{tabular}{|c|c|c|c|}
\hline & Aralbic & Irench & Russian \\
\hline Size of uncompressed list (MB) & 17 & 2.636 & 1 \\
\hline Ratio from a complete dictionary & 33 & 80 & 16 \\
\hline Number of inflected forms & 1.980 .280 & 247.406 & 75.234 \\
\hline Class decomposition (Prefixes) & 6 & 0 & 3 \\
\cline { 2 - 4 } & 13 & 84 & 23 \\
\hline (sutfixes) & 5660 & 892.646 & 348.636 \\
\hline $2-$ Mhysical compression & 4221 & 311.593 & 109.418 \\
\hline $3-$ FSM compression & 88 & 201.216 & 48.78 \\
\hline $4-$ Iogic compression & 253.686 & 480.770 & 163.202 \\
\hline $4+1$ & 145.086 & 207.376 & 56.784 \\
\hline $4+2$ & 121.500 & 104.665 & 37.74 \\
\hline $4+3$ & 57.214 & 150.321 & 36.71 \\
\hline
\end{tabular}

Table 2

weak inflexion factor, Russian which has a medium inflexion factor. lixperinents are done in two ways. First by using our logic compression alone and, then, in conjunction with other methods by supplying the redneed lexicon (list of compressed words in text format) obtained with ou method as input to existing methods. The three other methods tested are the following:

- Physical compression: Using a commercial physical process (Stuffit).

- Morpho-physical compression: This method was used to compress dictionaries used to build a spell checker [4]. It combines morphological proprieties by taking into account the suffixes of the language, but without any link between them. It also contains some physical features [7].

- ISM (Finite-State Machine) Compression: Using the Lexc (Finite State Lexicon Compiler) which allows the conversion of a list of surface forms into a transducer which is then minimized [8].

Results are described in table 2.

\section{IIr.4. Interpretations:}

The nost interesting thing observed on this table is the improvement obtained when we combine our method with a previons one. These results show that the existing methods are not optimal and can be improved by our logical compression in its first step. These important results in storage space should not hide others aspects of spell checker systems (retrieval and guessing). It woukd be interesting if the results given in the table were followed by other results showing improvements in the retrieval and gnessing of words.

\section{A PROPOSED ARCHITECTURE OF} A UNIVERSAL SPIELLING CHECKER:

Figure 3 shows the architecture of our proposed miversal spelling checker. Our method is inspired from previous methods ( $\$$ I.2), but presents some new original aspects that allow it to be considered a truly multilingual solution. In summary, our system has the following fealures:

- Multilinguism: this method will insure the multilingual constraint. By using different tools, specific to each language, to create a list of all surface forms.

- Storage space: by introducing the logic compression into the compression process, we will be able to get a reduced lexicon for whatever language we have to use. One task that still remains is to improve the logic compression by making the lask of linding the best decomposition more automatic. This problem is combinatorial; we must discover how 10 apply the optimization algorithms (genetic algorithm, stochast ic algorithm,...) in each case 10 find an optimal reduced lexicon starting from the large word-dictionary and primitive morphological knowledge (list of allixes and vowets).

- Retrieval/guessing: even lhough we haven't any concrele results now, the first experiments show that the process of checking words in an ISM formalism is faster that other existing methods. I 'urthermore, we are exploring paths to introluce functions (similarity $\mathrm{key}, .$. ) into the final obtained lexicon to make a rapid guessing of replacements for mistpelled words.

\section{CONCIJUSION}

Our approach to spell checking differs from previous methods by taking into account a new parameter which is

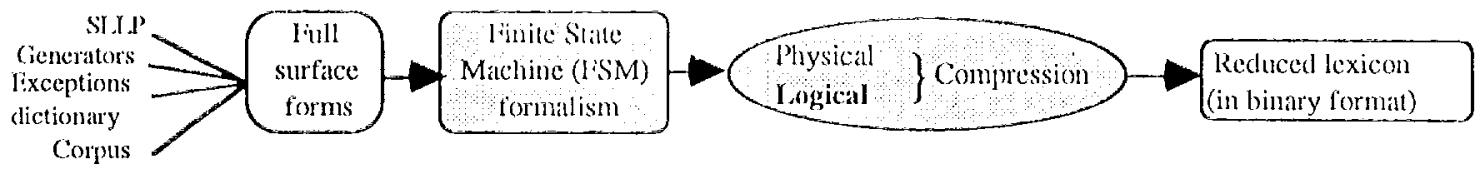

Fg. . . Universal sjelling checker 
the multilinguism. The system proposed tries to give solutions for the three main problems: Multilinguism, detection/guessing and storage size.

The first results, although using a manual method to find the decomposition in this first step, show that the previous methods to store dictionaries are not optimal and can be improved by exploring other techniques from the language itself. Another interesting experiment is to find an original optimization algorithm to find the optimal reduced lexicon that represents faithfully the initial list without any silence (missing words) or noise (incorrect words). Yet another project is to build a more robust method for the two olher problems (detection and guessing) from the reduced lexicon.

\section{ACKNOWLEDGMENTS}

The author would like to thank Prof. Christian BOITET for his constant support and encouragement. I am also very grateful to $\mathrm{Mr}$. Kenneth BEESI.EY (Rank Xcrox, Grenoble) for his fruitful discussions and Mr. Lauri KARTTUNIEN (Rank Xerox, Grenoble) for his help to realize some experiments.

\section{REFERENCES}

[1] Beesley K. R., Bukwalter T., (1989) Two-level, Finite-State Analysis of Arabic Morphology. Procecdings of the Seminar on Bilingual Computing in Arabic and English, 6-7 Sept. 1989. Cambridgc, England: The Literary and Linguistic Computing Center \& The Center for Middle Fastern Studies.

[2] Beesley K. R., (1990) Finite-state description of Arabic Morphology, in the Proceding of the Second Cambridge Conference on Bilingual Computing in Arabic and Inglish, Cambridge, Fingland, 6-7 September 1989. No pagination.

[3] Ben Mamadou A., (1986) A Compression technique for Arabic Dictionaries: The affix Analysis, in the Procceding of COLING-86, Bonn 1986, pp. 286-289.

[4] Circle Noetic Services (1989) Passwd, Reference Manual, MrT Branch Office, Boston, pp. 1-6.

[5] Circle Noetic Services (1989) Conjugate tool, Reference Manual, MIT Branch Office, Boston, pp. $1-5$.

[6] Glantz II., (1957) On the recognition of information with a digital computer, J. ACM, Vol. 4, No. $2,178-188$.

[7] Iuffman D. A., (1951) A method for the construction of minimum redundancy codes, Proc. IRL 40 (1951), 1098-1101.

[8] Karttunen L. (1993), Finite-Stale Lexicon Compiler, Xerox Palo Alto Research Center, April 1993, $1-35$.

[9] Koskeniemmi K., (1983) Two level Morphology, Publication no. 11, Department of Gencral Linguistics, University of Helsinki, pp. 18.

[10] Meddeb H.B., (1993) Intégration d'une composante morphologique pour la compression d'un diction- naire arabe, in Proc. Langue Arabe ot 'lechnologics Informatiques Avancées, Casablanca, pp. 14.

[11] Meddeb IY.X., (1994) Logic Compression of Multilingual dictionaries, in Proc. of ICIMCO-94 International Conference and Exhibition on Multi-lingual Computing, University of Cambridge, Center of Middle Eastem Sudics, London, April-1994, pp. 14.

[12] Oflaxer K, Solak A, (1992) Parsing agglutinative word structures and its application to spelling checking for Turkish, Proc. of COL.ING-92, Nantes, Aug. 23-28, vol. 1, pp. 39-45. 